\title{
El aprendizaje organizacional en instituciones de educación superior. Caso de estudio de un grupo de investigación
}

\author{
Organizational Learning in Higher Education Institutions. Case Study of a Research Group
}

\author{
Aprendizagem organizacional em instituições de ensino superior. Estudo de caso de um \\ grupo de pesquisa
}

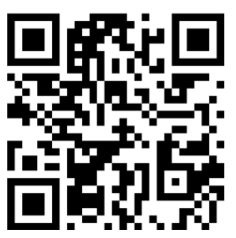

Recibido • Received • Recebido: 27 / 09 / 2019

Corregido • Revised • Revisado: 03 / 05 / 2021

Aceptado • Accepted • Aprovado: 26 / 05 / 2021

\begin{abstract}
Resumen:
Introducción. El conocimiento es considerado hoy en día como uno de los factores más importantes en las organizaciones, ya que este permite que estas reaccionen a los cambios de entorno, pues el establecer flujos al interior de ellas les permite ser más competitivas e innovadoras. Pero qué pasa cuando ese conocimiento se encuentra en una institución de educación, en la cual, a pesar de ser considerado su activo más importante, no se establecen las redes de conocimiento apropiadas para que estas estén en capacidad de responder a estos cambios, y se impide desarrollar procesos de transferencia tanto interna como externa. Este artículo es derivado de la tesis doctoral denominada Aprendizaje organizacional como mecanismo de transferencia de conocimiento en las instituciones de educación superior. Objetivo. Mostrar como los grupos de investigación universitarios se comportan como organizaciones que aprenden y busca identificar los factores que permitían desarrollar el aprendizaje. Metodología. Para ello, se diseñó un estudio de corte cualitativo, del tipo descriptivo-evaluativo, no experimental, de una sola medición, en la cual se empleó una triangulación de instrumentos que permitan corroborar y dar validez a los hallazgos a través del uso de herramientas como entrevistas, encuestas, observación participante y análisis de redes sociales. Resultados. Los resultados obtenidos fueron analizados con herramientas especializadas para datos cualitativos tales como MaxQDA y Ucinet en métodos de investigación mixtos. Conclusiones. El estudio permitió identificar cómo las instituciones de educación y, en particular, los grupos de investigación se comportan como organizaciones que aprenden y conforman redes de interacciones sociales que establecen diferentes tipos de flujos entre ellas.
\end{abstract}

Palabras claves: Aprendizaje organizacional; conocimiento; instituciones de educación superior; transferencia de conocimiento. 
http://doi.org/10.15359/ree.25-3.3

http://www.una.ac.cr/educare

educare@una.ac.cr

\begin{abstract}
:
Introduction. Knowledge is considered today as one of the most important factors in organizations since that allows them to react to changes in the environment, as the establishment of flows within them allows them to be more competitive and innovative. But what happens when that knowledge is in an educational institution, in which, despite being considered its most important asset, the appropriate knowledge networks are not established so that they can respond to these changes, preventing the development of internal and external transfer processes. This original article is derived from the doctoral thesis titled Organizational Learning as a Mechanism of Knowledge Transfer in Higher Education Institutions. Objective. To show how university research groups behave as learning organizations to identify the factors that allowed developing that learning. Method. For this, a qualitative study of a descriptive-evaluative, non-experimental, single-measure type was designed, in which a triangulation of instruments was used to corroborate and validate the findings through the use of tools such as interviews, surveys, participant observation, and analysis of social networks. Results. The results obtained were analyzed with specialized tools for qualitative data as MaxQDA and Ucinet used in mixed research methods. Conclusions. The study allowed identifying how education institutions and, in particular, research groups could become learning organizations and make social networks with different types of flows between them.
\end{abstract}

Keywords: Organizational learning; knowledge transfer; knowledge; higher education institutions.

\begin{abstract}
Resumo:
Introdução. O contato é considerado como um dos fatores mais importantes nas organizações, que permite que essas ações reajam aos índices de vendas, estabelecendo fluidos no interior da marca que permite ser mais competitivo e inovador. Pero qua pasa cuando ese conocimiento se encontrar en el institución de educación, no cual, um peso de ser considerado ativo mais importante, não estabelecer as redes de conocimiento apropiadas para que estas estejam em capacidade de responder a estos cambios, impedindo o arrolamento processos de transferência tanto interna como externa. Este artigo original, é derivado da tese de doutorado denominada Aprendizagem Organizacional como Mecanismo de Transferência de Pessoas em Instituições de Educação Superior. Objetivo. No caso em questão, como objeto de estúdio de estudos de grupos de investigação universitária como organizações que aprenderam com o objetivo de identificar os fatores que permitem o aprendizado da aprendizagem. Metodologia. Para ello, veja um estúdio de corte cualitativo, do tipo descritivoavaliativo, não experimental, de uma solução médica, no caso de uma triangulação de instrumentos que permita corroborar e dar validez a hallazgos através de uso de herramientes como entrevistas, entrevistas, observação participante e análises de redes sociais. Resultados. Os resultados obtidos são analisados com dados herdados utilizados para dados qualitativos e métodos de investigação mistos. Conclusão. O estúdio permite identificar como os institutos de educação e, em particular, os grupos de investigação que podem ser organizados por alunos.
\end{abstract}

Palavras-chave: Aprendizagem organizacional; conhecimento; instituição de educação superior; transferência de conhecimento. 
http://doi.org/10.15359/ree.25-3.3

\section{El conocimiento. Un acercamiento}

Son múltiples las definiciones que se tienen acerca del conocimiento, cada una de ellas con elementos comunes y algunos particulares. Para algunos, podría decirse que el conocimiento es el compendio de elementos entre los que se incluyen las experiencias, valores e información de las personas, además, elementos que sirven de marco de acción tal y como lo expresan Davenport y Prusak. (1998) para incorporar nuevo conocimiento. El conocimiento está ligado al ser y por ello las personas lo crean de forma racional o irracional, su complejidad obliga a ser estudiado por diferentes áreas y disciplinas tales como la filosofía, la epistemología y la gnoseología, así como también por la psicología y la hermenéutica entre otras, todas aportan a su definición y enriquecen el concepto de significados y significantes diferentes. En este caso nos abocamos al aprendizaje organizacional, orientado a la generación del conocimiento desde el trabajo colaborativo en un grupo de investigación.

Igualmente, Nonaka y Takeuchi (1999), tomando la clasificación epistemológica de (Polanyi, 1969), hablan de que el conocimiento se puede clasificar de modo tácito o explicito, que este también es personal y diferente en cada persona, además, no es posible explicarlo desde lógicas algorítmicas, dado que para su construcción es necesario involucrar las emociones humanas, lo que le proporciona un carácter de irracionalidad. Estos dos tipos de conocimiento se complementan y subyacen uno en el otro.

De acuerdo con Savage (1991), el conocimiento se ha convertido hoy en día en uno de los factores que generan riqueza para las organizaciones, sin embargo, la percepción sobre este ha cambiado históricamente. Resulta paradójico observar el hecho de cómo este inició siendo un atributo exclusivo de la nobleza y de la iglesia, sobre el cual se tenía un poder total y absoluto, empleado como un mecanismo de dominación y de opresión sobre las personas no letradas. Hoy ha llegado a convertirse en el motor que impulsa las sociedades, que permite su evolución, por el cual son valoradas las personas, que deben emplearlo en contexto social y sobre todo organizacional y que, además, las lleva a innovar.

En la ingeniería del conocimiento (Schreiber et al., 2000) se dice que el conocimiento es un cuerpo completo de datos e información que las personas pueden poner en práctica para desarrollar tareas y crear nueva información. Es decir, tiene un propósito de uso, es la maquinaria intelectual que permite alcanzar una meta. Adicionalmente, tiene una capacidad de generación porque su principal función es generar nueva información. No es, entonces, accidental que al conocimiento se le defina como el nuevo factor de producción.

Para esta misma área, Milton (2007) dice que el conocimiento está ligado a dominios de conocimiento, y que la experiencia que desarrollan las personas dentro de este dominio es la que les permite realizar eficientemente su trabajo, al buscar en él resolver situaciones complejas. 
http://doi.org/10.15359/ree.25-3.3

http://www.una.ac.cr/educare

educare@una.ac.cr

Tomando como base las anteriores definiciones, se retomó una construcción de Rodríguez Lora (2012) en la cual se incluyen los aspectos más importantes contenidos en estas:

El conocimiento es un conjunto de experiencias, conceptos, valores, creencias, relaciones conceptualesy sensaciones, que permitenal serhumano resolver problemasy desenvolverse exitosamente en un entorno, las cuales son producto de las interacciones del ser humano con su propio medio, y que para poder ser representados o modelados deben llevarse a un lenguaje formal, incluyendo en esta representación, las reglas de procedimiento y el modelo conceptual del dominio del conocimiento en particular. (pp. 17-18)

La forma en la que el conocimiento ha transformado al entorno, a la sociedad y a las organizaciones, ha hecho que este se vuelva en el objeto de estudio y que, precisamente, desde los estudios organizacionales exista una corriente dedicada a entender cómo los diferentes flujos de conocimiento impactan la operación de las organizaciones y permiten la creación de redes de confianza para el crecimiento de estas. El aprendizaje organizacional busca entender cómo el conocimiento es manejado al interior de estas y es puesto al servicio de los objetivos que esta se traza.

\section{Conocimiento en las organizaciones}

En los tiempos actuales, el conocimiento cobra un valor relevante dentro de la sociedad y en las organizaciones. El conocimiento que solo son capaces de generar las personas es el que permite que las organizaciones puedan dar respuesta a los cambios del medio, de crear y generar soluciones a las necesidades de los clientes y de la sociedad, de innovar mejorando los productos e impactando simultáneamente al entorno. Por ello, las organizaciones deben ver en su uso y creación una fuente generadora de ventajas competitivas que deben ser adecuadamente aprovechadas.

Entonces, el conocimiento es el motor que pone en funcionamiento a la sociedad, permite su evolución y que las organizaciones alcancen su objetivo de crecer y permanecer en el tiempo, y cuando logran esta meta deben ser vistas como organizaciones inteligentes, que aprenden, generan conocimiento, innovan, se adaptan y, sobre todo, transforman el entorno (Nonaka y Takeuchi, 1999).

Es así que el conocimiento también puede verse reflejado al interior de las organizaciones, las cuales, a su vez, son una representación de nuestras sociedades. Allí el entendimiento de cómo el conocimiento puede aportar a las organizaciones a su crecimiento es vital, dado que este ha permitido que se transformen, se reconfiguren e impacten el entorno. 
http://doi.org/10.15359/ree.25-3.3

Para lograr el objetivo de las organizaciones, el conocimiento y la ciencia debieron ponerse a su disposición, y estas debieron adoptar un nuevo estilo de producción en masa, que obligó a las grandes compañías simultáneamente a generar nuevos conocimientos. Allí, surgen interrogantes acerca de la forma en la que esta nueva industria debería ser gestionada, pues la producción no era la misma; los insumos deberían manejarse de un modo diferente; los obreros y obreras deberían crear nuevas tácticas de rendimiento en el trabajo; su trabajo y su conocimiento se hacía cada vez más especializado, se alineaba cada vez más con las realidades de la organización y crecía en ella y para ella. Se comienza a valorar el conocimiento técnico del personal operario, los conocimientos de tipo técnico permitían introducir cambios rápidos en los métodos de trabajo, pero son los de tipo social los que efectúan cambios sociales que permiten afrontar diferentes clases de situaciones (Mayo, 1977). De acuerdo con lo anterior, se puede evidenciar que hablar de conocimiento no es un tema nuevo en las organizaciones, se inicia desde las teorías de la administración científica hasta llegar a las teorías organizacionales del aprendizaje de hoy.

Es, entonces, cuando comienza a alinearse el conocimiento de los entes colaboradores organizacionales a la estrategia que esta ha definido, y es que, sería imposible desarrollarla sí el recurso humano que opera en ella no pone a disposición de la organización sus habilidades, técnicas, destrezas, conceptualizaciones científicas y el desarrollo de soluciones para que esta sea más productiva y eficiente. Y es, precisamente, el establecimiento de la estrategia lo que determina la forma en la que las organizaciones se transforman respecto a los cambios generados en la sociedad donde se encuentran inmersas, por lo cual es necesario que el conocimiento se ponga a disposición de estos propósitos, creando estructuras culturales que permitan que el conocimiento sea compartido y usado para facilitar que las organizaciones fluyan, innoven y crezcan.

$Y$ es principalmente en este punto donde se encuentra la mayor dificultad de las organizaciones y donde surge la pregunta sobre cómo crear estructuras culturales que faciliten que las personas pongan su conocimiento en beneficio de la organización y se sientan con el suficiente nivel de confianza que les permita hacerlo.

\section{Aprendizaje organizacional y transferencia de conocimiento}

Es factor fundamental del aprendizaje organizacional -AO en adelante- la capacidad que posee la organización que aprende a adaptarse al entorno (March y Olsen, 1975). Para ello este tipo de organizaciones debe asumir que debe estar en constante proceso de aprendizaje y creación de conocimiento y que, en ello, este aprendizaje exige, a su vez, que las organizaciones desarrollen la capacidad de transformar y modificar simultáneamente su entorno, generando en este proceso un ciclo continuo (Swieringa y Wierdsma, 1995). Por ello, el desarrollo de esta capacidad hace que las organizaciones puedan ser altamente innovadoras, y esto les permite generar mejores productos o servicios. 
http://doi.org/10.15359/ree.25-3.3

http://www.una.ac.cr/educare

educare@una.ac.cr

Sin embargo, es necesario hacer una diferenciación entre gestión del conocimiento -GC en adelante- y AO. De acuerdo con Davenport y Prusak (1998), el individuo es quien porta y posee el conocimiento, y es el encargado de la generación de los activos intangibles para la organización, además, responsable de ponerlos desde un enfoque gerencial en función de los procesos organizacionales para hacerlos más eficientes y productivos. Se habla entonces de $\mathrm{AO}$, cuando este conocimiento impacta la estructura, los procesos de toma de decisiones, y los esquemas relacionales de una organización como tal. La transición desde GC a AO exige, entonces, una dinámica del conocimiento en sí mismo, que debe fluir en la estructura organizacional, generando diferentes grados de interacción entre los individuos y el colectivo.

El objetivo de este $\mathrm{AO}$ es la generación de competencias que le permitan a la organización mejorar sus procesos y resultados de acuerdo con lo expresado por Chiva y Alegre (2005), y que, por ende, hace que el conocimiento sea resultado de un proceso organizacional.

El AO puede darse en las organizaciones a niveles individuales o grupales, y permite desarrollar competencias que son de valor para el entorno competitivo y las cuales pueden ser tomadas como factores diferenciadores, y que además pueden ser un factor determinante en el nivel de rendimientos y competitividad de la organización (Crossan et al., 1999). Entonces, la capacidad que desarrollan estas organizaciones y la velocidad a la que pueden aprender es la característica que se convierte en una ventaja competitiva sostenible, en la cual el desarrollo de dichas capacidades le permite a la organización garantizar su supervivencia.

Friedman (2003) determina que los individuos son fundamentales para propiciar el AO. Estos aportan sus conocimientos particulares en los procesos organizacionales impactando los resultados, su accionar permite que el conocimiento se transforme y permee a otros individuos. Sin embargo, tal y como lo exponen Alcover y Gil (2002), es necesario que para que este conocimiento se difunda, generar los medios desde el AO que permitan que el conocimiento se transforme desde lo individual a lo colectivo a través de la generación de reglas, prácticas, procedimientos, etc. Respecto a esto, Levitt y March (1988) proponen que estos conocimientos sean transmitidos a través de procesos de socialización, educación, profesionalización, imitación e intercambio.

Pero para que exista $\mathrm{AO}$, el conocimiento debe dejar de ser individual y transformarse en colectivo a través de lo indicado por Friedman (2003), donde la organización debe generar modelos mentales colectivos, haciendo que el conocimiento grupal generado a partir de estos esté en la capacidad de impactar los conocimientos particulares de los individuos. Este conocimiento es transformador, ya que para su construcción es necesario interpretar el entorno y responder a esas interpretaciones por medio de procedimientos y reglas organizacionales (Daft y Weick, 1984). La organización se torna, entonces, en un elemento decodificador del contexto, y el aprendizaje actúa como el proceso que lo permite y favorece que esta pueda operar.

6

Vanessa Rodríguez-Lora y Juan Manuel Herrera-Caballero

Los artículos de la Revista Electrónica Educare del Centro de Investigación y Docencia en Educación de la Universidad Nacional, Costa Rica, se comparten bajo términos de la Licencia Creative Commons: Reconocimiento, № Comercial, Sin Obra Derivada 3.0 Costa Rica. Las autorizaciones adicionales a las aquí delimitadas se pueden obtener en el correo: educare@una.cr 
http://doi.org/10.15359/ree.25-3.3

Para los autores Alcover y Gil (2002), la forma en la que se desarrolla el aprendizaje en las organizaciones está dada por un conjunto de descriptores y de guías que lo faciliten. Adicionalmente, exponen que cuando se habla de organizaciones que aprenden este conjunto de descriptores y guías es reemplazado por un conjunto de normas que hacen que el aprendizaje sea un deber ser en la organización. Esta caracterización está asociada a los conceptos de explotación y exploración, en donde el primero hace referencia al conocimiento ya existente en la organización y en su uso para garantizar la viabilidad diaria; mientras que el segundo hace referencia a la creación de nuevo conocimiento encaminado a la viabilidad futura.

Desde la perspectiva de Davenport y Prusak (1998), cuando las organizaciones realizan procesos efectivos de transferencia de conocimiento, la generación de ventaja competitiva les permite actualizar sus modelos y procesos de un modo menos costoso a través de la creación de redes con los generadores de conocimiento. Es allí donde estos autores afirman que en la consolidación de dichas redes hay beneficios para todos los actores involucrados, ya que, al hacer uso del conocimiento para la creación de uno nuevo, están innovando.

\section{Aprendizaje organizacional en instituciones de educación superior}

La importancia de estudiar hoy a las organizaciones que aprenden radica en la posibilidad de generación de capacidades de innovación, de productividad y de competitividad en la toma de decisiones y en el cambio organizacional que les permite reconfigurarse de acuerdo con los cambios del entorno; los cuales, según Nonaka (1994), facilitan en los individuos la creación de nuevo conocimiento, el cual no es visto como algo particular, sino como algo propio dentro de la misma organización, ya que se socializa en un proceso de generación de conocimiento compartido. Según este autor (Nonaka, 1994), en una economía cuya única certidumbre es la incertidumbre, la mejor fuente para obtener ventajas competitivas duraderas es el conocimiento.

Garzón Castrillón y Fisher (2008) indican que las organizaciones de hoy evidencian la importancia de desarrollar procesos de aprendizaje organizacional basándose en la creación y valoración del conocimiento que estas desarrollan, el cual se encuentra en las personas y que, con su interacción, han permitido el desarrollo de lo económico y de óptimos niveles de productividad. Esto se ha originado, además, por las capacidades que los entes colaboradores han desarrollado y que han puesto al servicio de las organizaciones de modo estratégico diferenciando a las organizaciones entre sí.

Ligado al tema de la creación de conocimiento que hace parte del aprendizaje organizacional, está entonces el de su transferencia hacia otros actores, lo que permitirá, a su vez, que estos también estén en capacidad de innovar (Simonen y McCann, 2010), incorporando este conocimiento a los procesos productivos de las organizaciones o a la toma de decisiones de las personas a las cuales se ha dirigido esta. Y es precisamente donde Bayona Sáez y Gonzalez Eransus (2010) muestran que existe transferencia que se origina principalmente desde las 
http://doi.org/10.15359/ree.25-3.3

http://www.una.ac.cr/educare

educare@una.ac.cr

universidades hacia el sector productivo, acorde con sus necesidades de fortalecimiento. Estas deben considerarse para las primeras un aspecto tan importante orientado desde la academia y la investigación; a diferencia de la transferencia adoptada a partir de las necesidades específicas de los clientes en las empresas.

El conocimiento que se genera en las universidades a través de los diferentes procesos que esta realiza es llevado al sector organizacional, para que sea incorporado en este y generar, desde allí, un mayor desarrollo operativo y económico de las organizaciones.

Aquí cobra importancia el conocimiento desarrollado en las universidades a través de sus procesos de I+D, o de I+D+i; este les permite responder a las necesidades y requerimientos del entorno, pero también a impactarlo al hacer una transferencia de lo aprendido. De acuerdo con Macho-Stadler (2010), las instituciones de educación superior han creado internamente tecnologías importantes, así como también conceptos y valores sociales que hacen, por lo tanto, necesario que estos sean llevados al entorno general a través de los diferentes grupos de interés.

Para Bayona Sáez y González Eransus (2010), la transferencia realmente se da en dos aspectos: Transferencia de conocimiento, representada por el conocimiento producido al interior de las universidades y llevado al entorno por medio de capacitaciones, sensibilizaciones, planes de emprendimiento y adopción de modelos. Transferencia de tecnológica, cuando el conocimiento producido al interior puede llevarse a las organizaciones, representada en tecnología que puede patentarse.

Sin embargo, es paradójico observar que los procesos de trasferencia de conocimiento e incluso aprendizaje organizacional son incipientes o inexistentes al interior de este tipo particular de organización. Y es que, al contrario de lo que se piensa, son pocas las prácticas existentes en este sentido que se han logrado oficializar dentro de las instituciones de educación superior.

\section{Desarrollo metodológico}

El estudio realizado para determinar los factores que permiten el desarrollo del aprendizaje organizacional, y que este a su vez se presente como un mecanismo de transferencia de conocimiento, fue de corte cualitativo, en una investigación descriptiva evaluativa, no experimental, de una sola medición, en la cual los datos fueron corroborados a partir de métodos de triangulación.

Se seleccionó como objeto de estudio al grupo de investigación de Ciencias Administrativas del Instituto Tecnológico Metropolitano- ITM en Medellín, Colombia. Este grupo fue seleccionado debido a que ha presentado un desarrollo acelerado respecto a las mediciones de grupos que ha realizado en el país COLCIENCIAS (Departamento Administrativo deCiencia, Tecnología e Innovación), donde en un corto periodo de tiempo pasó a ostentar la categoría más alta dada en el país.

Vanessa Rodríguez-Lora y Juan Manuel Herrera-Caballero

Los artículos de la Revista Electrónica Educare del Centro de Investigación y Docencia en Educación de la Universidad Nacional, Costa Rica, se comparten bajo términos de la Licencia Creative Commons: Reconocimiento, No Comercial, Sin Obra Derivada 3.0 Costa Rica. Las autorizaciones adicionales a las aquí delimitadas se pueden obtener en el correo: educare@una.cr 
http://doi.org/10.15359/ree.25-3.3

El modelo de medición de grupos de COLCIENCIAS define diferentes categorías desde su reconocimiento como grupo creado hasta una categoría denominada $A 1$, en la cual el grupo destaca por su impacto en las publicaciones, el personal investigador que la conforma, el talento humano formado y la generación de nuevo conocimiento. Este grupo en particular logro la más alta categoría en un periodo no superior a cuatro años.

Para la obtención de los datos, se aplicaron instrumentos que permitieran obtener resultados mixtos tales como entrevistas, encuestas, observación participante, grupos focales, entre otras, y para su análisis se emplearon analizadores semánticos como MaxQDA y de relaciones en redes sociales como Unicet. El grupo estaba constituido por 38 personas docentes investigadoras, agrupadas en tres líneas de investigación.

\section{Hallazgos}

El estudio realizado evidenció que, si bien es cierto que existen procesos definidos de transferencia tanto de tecnología como de conocimiento hacia el entorno organizacional, no hay mecanismos para generar flujos de conocimiento hacia el interior de la misma institución, lo cual es lo que da sustento al aprendizaje organizacional, y que denota que aunque el conocimiento es el activo organizacional más importante para las instituciones de educación superior, no se han desarrollado estrategias que permitan su intercambio al interior de esta.

Un ejemplo de lo anterior se manifiesta a través del desarrollo de proyectos con temáticas similares por diferentes grupos o personal de investigación, y que podrían obtener mejores resultados con la conformación de grupos inter y multidisciplinares, los cuales desde sus particulares campos de actuación podrían dar origen a productos mucho más enriquecidos.

Si bien no existen mecanismo oficialmente constituidos enfocados en el desarrollo de aprendizaje organizacional, los actores del proceso de investigación sí denotan la importancia de consolidarlos, de que se generen flujos que permitan que el conocimiento pase de los niveles personales a grupales y que, a su vez, estos puedan impactar en la institución en la que están inmersos.

Y es en este punto donde se encuentra la principal crítica a los procesos relacionados con la generación de conocimiento en las organizaciones, ya que el crecimiento en su desarrollo es elevado, pero el impacto que este tiene en los procesos académicos y misionales es mínimo. En cuanto al cumplimiento de indicadores de medición prima, no hay mecanismos que permitan que el conocimiento se difunda, se transmita y transforme la academia.

Para poder entender, entonces, este fenómeno, es necesario recurrir a los aportes que desde otras ciencias alimentan el análisis que se viene desarrollando, tal es el caso del psicoanálisis y sociología organizacional. Y es que es claro cómo algunos individuos dentro de las organizaciones modifican sus conductas y su modo de comportamiento a fin de lograr un 
http://doi.org/10.15359/ree.25-3.3

http://www.una.ac.cr/educare

educare@una.ac.cr

acoplamiento y generar un sentido de identidad y pertenencia. Pero es precisamente este tipo de comportamiento el que es ideal dentro de la generación de flujos de conocimiento al interior de las instituciones; los individuos solo estarán en disposición de poner su conocimiento en función de los intereses de la organización, si esos intereses colectivos hacen parte también de sus intereses particulares. Además, estos flujos de conocimiento se generan dentro de la organización solo cuando los individuos están en disposición de aportar con su conocimiento al logro de los objetivos. Según Crossan et al. (1999, citados por Rodríguez-Lora, 2019, p. 34), estos flujos son de dos tipos, "tales como los flujos de explotación que permiten la continuidad organizacional y los flujos de exploración que son los que promueven el cambio. Estos son entonces los encargados de dinamizar el conocimiento en la organización". Pero es necesario recalcar que los individuos estarán en disposición de aportar solo en la medida que ello les implique un beneficio personal, que los haga crecer económica o profesionalmente, como lo explican de Gaulejac et al. (2006) cuando tratan de determinar cuáles son los mecanismos que articulan estos comportamientos individuales con los colectivos, cuando analizan cómo los seres humanos establecen relaciones dentro de los lugares sociales, en este caso en particular, la organización que aprende, tal como lo deberían ser las instituciones de educación superior junto con sus procesos de investigación.

Es necesario entender que estas interacciones, particularmente las relacionadas con el uso del conocimiento, no siempre se desarrollan de manera consciente al interior de los individuos. Muchos sujetos investigadores solo buscan el desarrollo de sus metas personales y, para ello, ponen su conocimiento en función de unos objetivos que se hacen colectivos. Esto puede explicarse, por ejemplo, en el caso de una institución de educación superior, por el hecho de que muchos docentes desarrollan productos de conocimiento tales como artículos, ponencias, patentes, etc., no pensando exclusivamente en los objetivos de posicionamiento que pretende la institución, sino que se encuentran enlazados sus intereses de reconocimiento particular dentro de la sociedad académica y científica. El posicionamiento de la institución, entonces, se da por transitividad de estos intereses netamente particulares.

Y es aquí donde las instituciones tienen que ser inteligentes en el desarrollo de estrategias que permitan que esos intereses particulares de los individuos se transformen en beneficios para ella. La gran pregunta será siempre, por tanto, ¿cómo generar una cultura organizacional en la cual los individuos no se sientan temerosos de compartir aquello que saben, ya que existe un beneficio tanto personal como organizacional detrás de ello?

Es importante que las organizaciones creen ambientes de confianza, donde culturalmente compartir el conocimiento sea una constante y permita que el conocimiento de los individuos se haga parte de la colectividad, y que este, a su vez, facilite la generación de nuevo conocimiento que dé respuesta a las necesidades del entorno en el cual está inmerso, un entorno que se encuentra en constante cambio y que obliga a estar preparado para los cambios que se manifiesten. 
http://doi.org/10.15359/ree.25-3.3

Es así como también, desde la construcción social de una realidad organizacional, el individuo puede sentirse identificado con su contexto $y$, por ende, con su realidad, lo que permite que elaboren una identidad con la organización y se conviertan en parte del colectivo al hacer que sus intereses se alineen con los intereses de esta. La forma en la que una organización habla, cómo define su cultura, sus modelos y arquetipos mentales, se hace parte del mismo individuo y permite que sus propios modelos se ajusten a los de esta. Esto quiere decir que la misma capacidad intelectual de los individuos es la que les permite ajustarse a las nuevas realidades que viven y desarrollan.

El mismo principio se aplica a las instituciones educativas, las cuales son, en su estructura funcional, organizaciones; pero, con una característica particular: generan y divulgan conocimiento, solo que pocas veces emprenden iniciativas reales que les permitan gestionarlo y aprender de él.

\section{Conclusiones}

Este artículo constituye parte de un estudio más complejo, en el cual se desean determinar cuáles son los factores que facilitan que las instituciones de educación superior realicen procesos de transferencia tanto internos como externos y constituyan flujos de conocimiento efectivo que faciliten su aprendizaje.

Si bien es cierto, para este tipo de instituciones, el conocimiento se toma como el principal factor productivo y facilitador de la prestación de su servicio de educación, este no fluye de manera adecuada en su interior; no existen unos procesos definidos de lo que debe considerarse como una transferencia de conocimiento interna $y$, por ende, no es posible establecer una estrategia de transferencia externa.

Para este estudio en particular, se ha tomado como referencia un grupo de investigación sobre el cual se ha podido identificar que no existe un proceso de transferencia formal o informal en su interior, donde el personal docente investigador no tiene información sobre los desarrollos temáticos que se están haciendo de manera global en la institución; ello hace que se realicen sobreesfuerzos en la generación de conocimiento que ya existe al interior; no hay un uso eficiente de los recursos de capital de humano y de conocimiento dentro del grupo, ya que no existe un inventario de la base de conocimiento grupal.

Tal y como se ha expresado anteriormente en este artículo, uno de los aspectos más importantes a desarrollar al interior de las organizaciones es el establecimiento de las redes de aprendizaje que sustentarán los diferentes flujos de conocimiento que, en sí mismos, permiten que la organización aprenda y que simultáneamente pueda hacer una transferencia de este conocimiento creado. Será tarea de futuras investigaciones proponer la forma de generar los flujos y cómo replicar modelos en las organizaciones de tal forma que se genere un aprendizaje y se constituyan organizaciones que aprendan. 
http://doi.org/10.15359/ree.25-3.3

http://www.una.ac.cr/educare

educare@una.ac.cr

Este estudio, en una fase más detallada del desarrollo metodológico de esta investigación, pretende identificar cuáles son los factores facilitadores o limitadores para desarrollar, conjuntamente, los procesos de aprendizaje y de transferencia, que le permitan a esta responder ante la realidad de la organización y del entorno que será el receptáculo final del conocimiento que esta esté en capacidad de generar.

\section{Declaración de Material complementario}

Este artículo tiene disponible, como material complementario:

-La versión preprint del artículo en https://doi.org/10.5281/zenodo.4042003

\section{Referencias}

Alcover, C. M. y Gil, F. (2002). Crear conocimiento colectivamente: Aprendizaje organizacional y grupal. Psicologia del Trabajo y de las Organizaciones, 18(2-3), 259-301. https://www. redalyc.org/articulo.oa?id=231318274008

Bayona Sáez, C. y González Eransus, R. (2010). La transferencia de conocimiento en la Universidad Púbica de Navarra. Una visión desde la empresa y desde el ámbito universitario. UPNA. https:// www.unavarra.es/investigacion

Chiva, R. y Alegre, J. (2005). Organizational learning and organizational knowledge: Towards the integration of two approaches. Management learning, 36(1), 49-68. https://doi. org/10.1177/1350507605049906

Crossan, M. M., Lane, H. W. y White, R. E. (1999). An organizational learning framework: From intution to institution. Academy of Management Review, 24(3), 522-537. https://doi. org/10.2307/259140

Daft, R. L. y Weick, K. E. (1984). Toward a model of organizations as interpretatio systemes. Academy of Management Review, 9(2) 284-295. https://doi.org/10.2307/258441

Davenport, T. H. y Prusak., L. (1998). Working knowledge. How organizations manage what they know. Hardvard Business School Press.

de Gaulejac, V., Rodríguez Márquez, S. y Taracena Ruiz, E. (2006). Historia de vida. Psicoanálisis y sociología clinica. Universidad Autónoma de Queretaro.

Friedman, V. J. (2003). The individual as agent or organizational learning. En M. Dierkes, A. Berthoin Antal, J.Child ye I. Nonaka (Eds.), Handbook of organizational learning \& knowledge (pp. 398-414). Oxford University press.

Garzón Castrillón, M. A. y Fisher, A. L. (2008). Modelo teórico de aprendizaje organizacional. Pensamiento y Gestión, (24), 195-224. http://www.scielo.org.co/scielo.php?script=sci artt ext\&pid=S1657-62762008000100008 
http://doi.org/10.15359/ree.25-3.3

http://www.una.ac.cr/educare educare@una.ac.cr

Levitt, B. y March, J. G. (1988). Organizational learning. Annual review of sociology, 14, 319-338. https://doi.org/10.1146/annurev.so.14.080188.001535

Macho-Stadler, I. (2010). Transferencia de las innovaciones universitarias. Els Opuscles del CREI, (25), 1-21. https://crei.cat/opuscles/

March, J. G. y Olsen, J. P. (1975). The uncertainty of the past: Organizational learning under ambiguity. European Journal of Political Research, 3(2), 147-171. https://doi. org/10.1111/j.1475-6765.1975.tb00521.x

Mayo, E. (1977). El peor aspecto del progreso. En E. Mayo (Autor), Problemas sociales de una civilización industrial (pp. 21-52). Ediciones Nueva Visión.

Milton, N. R. (2007). Knowledge acquisition in practice. A step-by-step guide. Springer.

Nonaka, I. (1994). A dynamic theory of organizational knowledge creation. Organization Science, 5(1), 14-37. https://doi.org/10.1287/orsc.5.1.14

Nonaka, I. y Takeuchi, H. (1999). La organización creadora de conocimiento: Cómo las compañías japonesas crean la dinámica de la innovación. Oxford University Press.

Polanyi, M. (1969). Knowing and being. University of Chicago Press.

Rodríguez Lora, V. (2012). Diseño e implementación de un modelo de conocimientos sobre las cuencas petrolíferas de Colombia [Tesis de maestría]. Universidad EAFIT. https://www. yumpu.com/es/document/read/14370281/diseno-e-implementacion-de-un-modelode-conocimientos-sobre-las-

Rodríguez-Lora, V. (2019). Capítulo 2. Los grupos de investigación vistos como organizaciones que aprenden. En G. Ramírez Martínez, J. M. Herrera Caballero, P. C. Solís Pérez y G. Vargas Laroos (Coords.), El análisis organizacional en el desarrollo local. Estudio de caso del entramado institucional y organizacional de Medellín, Colombia (pp. 32-48). Centro de Investigación y Desarrollo Ecuador.

Savage, C. (1991). The international trade show for digital equipment corporation. Presentación alDECWORLD. The International Trade Show For Digital Equipment Corporation, Presentación al DECWORLD. Boston, Massachusetts.

Schreiber, G., Akkermans, H., Anjewierden, A., de Hoog, R., Shadbolt, N., van de Velde,W.yWielinga, B. (2000). Knowledge engineering and management. The commonKADS methodology. MIT. https://doi.org/10.7551/mitpress/4073.001.0001

Simonen, J. y McCann, P. (2010). Knowledge transfers and innovation: The role of labour markets and R\&D co-operation between agents and institutions. Papers in Regional Science, 89(2), 295-309. https://doi.org/10.1111/j.1435-5957.2010.00299.x

Swieringa, J. y Wierdsma, A. (1995). La organización que aprende. Addison-Wesley. 